\title{
FRAGILITY FRACTURES AS THE INITIAL MANIFESTATION OF INDOLENT SYSTEMIC MASTOCYTOSIS
}

\author{
E. Vassilatou ${ }^{1}$, N. Gkavogiannakis ${ }^{3}$, A. Chatzipetrou ${ }^{3}$, Ch. Koulias 3 , M. Makris 3 , E. Garoflos ${ }^{1}$, D. Hadjidakis ${ }^{1,2}$, \\ D. Rigopoulos ${ }^{4}$, G. Dimitriadis ${ }^{2}$
}

${ }^{1}$ Endocrine Unit, ${ }^{2} 2$ nd Department of Internal Medicine-Propaedeutic, ${ }^{3}$ Mastocytosis Outpatient Clinic, Allergy Unit "D. Kalogeromitros", ${ }^{4} 2^{\text {nd }}$ Department of Dermatology and Venereology, "Attikon" University Hospital, Athens, Greece

\section{INTRODUCTION}

Systemic mastocytosis (SM) is a rare disease $(2$ cases per 100.000 population/ year) characterized by clonal proliferation of abnormal mast cells in several tissues, most often skin and bone marrow. Indolent systemic mastocytosis (ISM) is the commonest disease variant in adults, characterized by very low rate of mast cell proliferation. SM has been recognized as a cause of secondary osteoporosis.

\section{OBJECTIVE}

To evaluate bone mineral density and fragility fractures in ISM patients.

\section{METHODS}

Fourteen patients (9 women, 7 premenopausal), aged 27-63 years, diagnosed with ISM according to World Health Organization criteria (2008) were studied retrospectively.

Clinical examination, biochemical evaluation and bone mineral density (BMD) measurements by dual-energy $X$-ray absorptiometry at the lumbar spine $\left(L_{1}-L_{4}\right)$, the total proximal femur, the femoral neck and the distal one-third radius were performed.

T-score was used to define osteopenia (<-1 to $>-2.5$ SD) or osteoporosis (-2.5 SD or lower) in postmenopausal women or men aged 50 years or older, and z-score $\leq-2.0$ for low BMD in younger men and premenopausal women, according to the guidelines of International Society for Clinical Bone Densitometry. Fractured vertebrae were excluded from BMD measurement.

No patient reported other diseases or use of treatments known to affect bone or mineral metabolism, at initial assessment.

Table 1: Clinical characteristics of ISM patients.

\begin{tabular}{|c|c|c|c|c|c|c|}
\hline $\begin{array}{c}\text { Patient } \\
\text { No }\end{array}$ & Sex & $\begin{array}{c}\text { Age }\left(^{*}\right) \\
(\text { years })\end{array}$ & $\begin{array}{c}\text { BMI } \\
\left(\mathrm{Kg} / \mathrm{m}^{2}\right)\end{array}$ & Smoking & $\begin{array}{c}\text { Alcohol } \\
\text { intake }\end{array}$ & $\begin{array}{c}\text { Main symptom for SM } \\
\text { investigation }\end{array}$ \\
\hline 1 & F & 41 & 23.4 & Yes & Minimal & Urticaria pigmentosa \\
\hline 2 & F & 31 & 23.7 & Yes & Minimal & Urticaria pigmentosa \\
\hline 3 & F & 56 & 30.8 & No & Minimal & Skin lesions \\
\hline 4 & F & 43 & 33.5 & Yes & Minimal & vertebral fracture \\
\hline 5 & F & 27 & 22.3 & No & Minimal & Urticaria pigmentosa \\
\hline 6 & F & 63 & 24.9 & Yes & Minimal & Drug anaphylaxis \\
\hline 7 & F & 41 & 31.2 & No & No & Urticaria pigmentosa \\
\hline 8 & F & 45 & 31.6 & Yes & No & Anaphylactic shock \\
\hline 9 & F & 31 & 23.5 & Yes & Minimal & Rib fractures \\
\hline 10 & M & 38 & 26.6 & Yes & Minimal & Vertebral fracture \\
\hline 11 & M & 57 & 31.2 & Yes & Minimal & Urticaria pigmentosa \\
\hline 12 & M & 37 & 26.3 & No & Minimal & Food anaphylaxis \\
\hline 13 & M & 45 & 39.0 & No & Minimal & Skin lesions \\
\hline 14 & M & 53 & 31.5 & No & Minimal & Urticaria pigmentosa \\
\hline
\end{tabular}

(*) Age at initial bone mineral density evaluation

\section{RESULTS}

* Three patients $(21.4 \%)$ had fragility fractures: a 43-year-old premenopausal woman (patient No 4) \& a 38-year-old man (patient No 10) had vertebral fractures, while a 31-year-old premenopausal woman (patient No 9) had non-vertebral fractures (Table 1).

* Fragility fractures were the cause for SM investigation since none of these patients had cutaneous mastocytosis and only 1 patient reported a mild episode of anaphylaxis, at diagnosis (Table 1).

- $25(\mathrm{OH}) \mathrm{D}_{3}$ deficiency $(<20 \mathrm{ng} / \mathrm{ml})$ was detected in 7 patients $(50 \%)$ (Table 2).

* Bone densitometry showed osteoporosis in 2 patients $(14.3 \%)$, osteopenia in $2(14.3 \%)$ and low BMD in $7(50 \%)$ (Table 3). BMD zscore was generally lower at the spine than at the hip and at the femoral neck (all patients) $(p<0.001)$.

* Serum tryptase levels were negatively correlated $(r=-0,615, p=$ 0,019 with lumbar spine BMD z-score (all patients) (Figure 1).
Table 2: Laboratory characteristics of ISM patients.

\begin{tabular}{|c|c|c|c|c|c|c|c|c|c|c|}
\hline No & $\begin{array}{l}\text { tryptase } \\
(\mathrm{ng} / \mathrm{ml})\end{array}$ & \begin{tabular}{|c|} 
PTH \\
$(p g / m l)$
\end{tabular} & $\begin{array}{c}\mathrm{Ca} \\
\text { serum } \\
\text { (mg/dl) }\end{array}$ & $\begin{array}{c}\mathrm{P} \\
\text { serum } \\
(m g / d l)\end{array}$ & $\begin{array}{c}\text { creatinine } \\
(m g / d l)\end{array}$ & \begin{tabular}{|} 
Albumin \\
$(\mathrm{mg} / \mathrm{dl})$
\end{tabular} & $\begin{array}{c}25(\mathrm{OH}) \mathrm{D}_{3} \\
(\mathrm{ng} / \mathrm{ml})\end{array}$ & $\begin{array}{l}\text { ALP } \\
(U / L)\end{array}$ & $\begin{array}{c}\text { TSH } \\
(\mu / U / m)\end{array}$ & $\begin{array}{l}\text { Testo } \\
(\mathrm{mg} / \mathrm{d} / \mathrm{l})\end{array}$ \\
\hline 1 & 29.8 & 24 & 9.3 & 3.3 & 0.8 & 4.1 & 24.3 & 54 & 0.4 & \\
\hline 2 & 20 & 37.4 & 8.8 & 3.4 & 0.5 & 4.2 & 19.1 & 57 & 1.9 & \\
\hline 3 & 123 & 43 & 9.2 & 4.4 & 0.7 & 4.7 & 30.4 & 69 & 3.2 & \\
\hline 4 & 47.3 & 32 & 9.5 & 3 & 0.7 & 4.3 & 22.3 & 66 & 1.4 & \\
\hline 5 & 86.8 & 40,2 & 9.3 & 4.6 & 0.7 & 4.8 & 19.8 & 78 & 1.7 & \\
\hline 6 & 24.5 & 48.4 & 9.2 & 3.9 & 0.7 & 4.3 & 20.8 & 64 & 1.1 & \\
\hline 7 & 57 & 38.2 & 9.3 & 2.5 & 0.6 & 3.9 & 9 & 95 & 1.3 & \\
\hline 8 & 94.7 & 21 & 9.4 & 3.7 & 0.7 & 4 & 17.7 & 65 & 1.2 & \\
\hline 9 & 17.4 & 24 & 9.1 & 4.3 & 0.6 & 4.3 & 28 & 55 & 1.8 & \\
\hline 10 & 70 & 34 & 9.2 & 3.1 & 1.1 & 4.7 & 27.7 & 92 & 1.2 & 330 \\
\hline 11 & 85 & 49 & 9.4 & 3.2 & 1 & 4.5 & 30 & 104 & 1.2 & 339 \\
\hline 12 & 35.5 & 31 & 9.5 & 4.2 & 0.8 & 4.9 & 12.5 & 62 & 1.8 & 425 \\
\hline 13 & 55.4 & 26.7 & 10.1 & 3.4 & 0.9 & 4.6 & 18.2 & 91.6 & 2.4 & 496 \\
\hline 14 & 26 & 33 & 10.2 & 4.1 & 1 & 4.8 & 16.7 & 81 & 0.8 & 446 \\
\hline
\end{tabular}

Figure 1: Correlation between serum tryptase levels \& LS BMD z-score

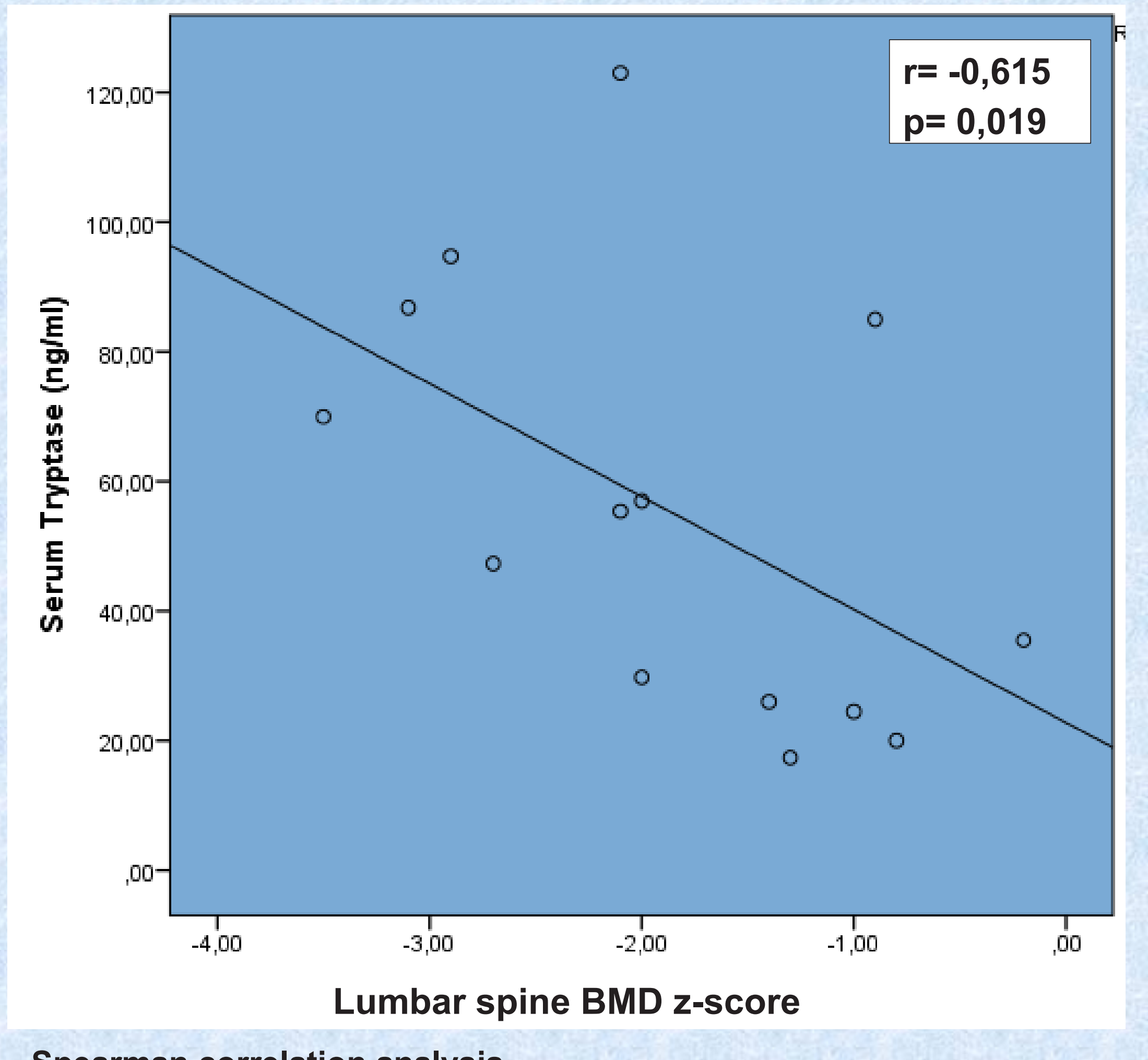

Table 3: Fracture history and BMD of ISM patients.

\begin{tabular}{|c|c|c|c|c|c|c|}
\hline No & $\begin{array}{l}\text { Non Frag } \\
\text { fractures }\end{array}$ & $\begin{array}{c}\text { Frag } \\
\text { fractures }\end{array}$ & $\begin{array}{l}\text { LS BMD }\left(^{*}\right) \\
\text { z/T-score }\end{array}$ & \begin{tabular}{|c|} 
T Hip BMD \\
z/T-score
\end{tabular} & $\begin{array}{l}\text { FN BMD } \\
\text { z/T-score }\end{array}$ & $\begin{array}{c}1 / 3 \text { radius } B M D \\
z / T \text {-score }\end{array}$ \\
\hline 1 & 0 & 0 & $z-2.1$ & z 0.6 & $z-0.2$ & z 1.5 \\
\hline 2 & left forearm & 0 & $z-0.8$ & $z-0.1$ & $z-0.4$ & $z-0.1$ \\
\hline 3 & left forearm & 0 & $\mathrm{~T}-3.4$ & T 0.1 & T 0.3 & $\mathrm{~T}-2.3$ \\
\hline 4 & 0 & \begin{tabular}{|c|} 
vertebral \\
fracture (L4)
\end{tabular} & $z-2.7$ & $z-0.8$ & z 0.2 & z 1.1 \\
\hline 5 & 0 & 0 & $z-3.1$ & $z-0.9$ & $z-1.1$ & $z-0.8$ \\
\hline 6 & 0 & 0 & $\mathrm{~T}-2.6$ & $\mathrm{~T}-1.6$ & $\mathrm{~T}-2.0$ & $\mathrm{~T}-0.1$ \\
\hline 7 & 0 & 0 & $z-2.1$ & $z-0.5$ & $z-0.8$ & z 1.1 \\
\hline 8 & $\begin{array}{c}\text { pelvis, right } \\
\text { forearm }\end{array}$ & 0 & $z-2.9$ & $z-1.3$ & $z-1.9$ & z 0.3 \\
\hline 9 & right forearm & Rib fractures & $z-1.3$ & $z-0.8$ & $z-1.6$ & $z-0.3$ \\
\hline 10 & 0 & $\begin{array}{c}\text { vertebral } \\
\text { fracture (L5) }\end{array}$ & $z-3.5$ & $z-0.9$ & $z-1.4$ & $z-0.3$ \\
\hline 11 & 0 & 0 & $\mathrm{~T}-1.6$ & $\mathrm{~T}-0.6$ & $\mathrm{~T}=1.5$ & $T-0.1$ \\
\hline 12 & 0 & 0 & $z-0.2$ & $z-0.5$ & $z-1.2$ & z 0.1 \\
\hline 13 & 0 & 0 & $z-2.2$ & z 0.3 & z 0.5 & $z-0.1$ \\
\hline 14 & $\begin{array}{l}\text { vertebral } \\
\text { fracture (L2) }\end{array}$ & 0 & $\mathrm{~T}-1.8$ & $\mathrm{~T}-1.3$ & $\mathrm{~T}-1.2$ & $\mathrm{~T}-1.9$ \\
\hline
\end{tabular}

Frag fractures: fragility fractures, LS: lumbar spine (L1-L4), T Hip: total hip, 1/3 radius: dista one-third radius, $\square$ denotes osteoporosis, $\square$ denotes osteopenia, $\square$ denotes low bone mass $\left.{ }^{*}{ }^{*}\right)$ Fractured vertebrae were excluded from BMD measurement.

\section{CONCLUSION}

Bone involvement is frequent in ISM patients and may be the initial manifestation. Osteoporotic fractures of unknown aetiology should lead to the suspicion of SM particularly in individuals younger than $\mathbf{5 0}$ years.

\section{REFERENCES}

Rossini $\mathrm{M}$ et al. Bone mineral density, bone turnover markers and fractures in patients with indolent systemic mastocytosis. Bone 2011;49:880

van der Veer $\mathrm{E}$ et al. High prevalence of fractures and osteoporosis in patients with indolent systemic mastocytosis. Allergy 2012: 67; 431

Guillaume $\mathrm{N}$ et al . Bone complications of mastocytosis: a link between clinical and biologica characteristics. Am J Med. 2013: 126; 75.e1 34: 383 